\title{
Cogitare
Enfermagem
}

\section{O ENFERMEIRO NOS PROGRAMAS DE GERENCIAMENTO DO USO DE ANTIMICROBIANOS: REVISÃO INTEGRATIVA}

\author{
Adriana Maria da Silva Felix', Sandra Regina Toffolo²
}

\section{RESUMO}

Objetivo: investigar na literatura publicações acerca da participação do enfermeiro nos programas de gestão do uso de antimicrobianos.

Método: revisão integrativa com busca nas bases de dados: Medical Literature Analysis and Retrieval System on Line, Literatura Latino-Americana de Ciências da Saúde e Cumulative Index to Nursing and Allied Health Literature. Como critérios de inclusão, foram selecionados artigos disponíveis na íntegra, publicados entre janeiro de 2007 e maio de 2018, nos idiomas português, inglês ou espanhol.

Resultados: foram encontrados 140 artigos, 12 atenderam aos critérios de inclusão, e foram organizados em três categorias: "competências do enfermeiro nos programas de gestão do uso de antimicrobianos" (cinco artigos), "déficit de conhecimento e necessidade de capacitação" (seis artigos) e "ação sistêmica" (um artigo).

Conclusão: os enfermeiros podem contribuir de forma substancial com os Programas de Gerenciamento do uso de Antimicrobianos, porém são necessárias ações educacionais, pesquisas e políticas públicas para que a sua participação seja efetiva.

DESCRITORES: Farmacorresistência Bacteriana Múltipla; Enfermeiro; Gestão de Antimicrobianos; Competência Profissional; Revisão Sistemática.

COMO REFERENCIAR ESTE ARTIGO:

Felix AM da S, Toffolo SR. O enfermeiro nos programas de gerenciamento do uso de antimicrobianos: revisão integrativa. Cogitare enferm. [Internet]. 2019 [acesso em "colocar data de acesso, dia, mês abreviado e ano"]; 24. Disponível em: http://dx.doi.org/10.5380/ce.v24i0.59324.

\section{(c) (1)}

Este obra está licenciado com uma Licença Creative Commons Atribuição 4.0 Internacional.

${ }^{1}$ Enfermeira. Doutora em Ciências. Docente de Enfermagem da Faculdade de Ciências Médicas da Santa Casa de São Paulo. São Paulo, SP, Brasil. ()

Enfermeira. Doutora em Ciências. Docente da Universidade Federal de Uberlândia. Uberlândia, MG, Brasil. () 


\title{
PARTICIPATION OF NURSES IN ANTIMICROBIAL STEWARDSHIP PROGRAMS: AN INTEGRATIVE REVIEW
}

\author{
ABSTRACT \\ Objective: To investigate publications in the literature on the participation of nurses in \\ antimicrobial stewardship programs. \\ Method: Integrative review with search in the following databases: the Medical Literature \\ Analysis and Retrieval System Online, the Latin American and Caribbean Center on Health \\ Sciences Information, and the Cumulative Index to Nursing and Allied Health Literature. As \\ inclusion criteria, full articles in Portuguese, English, or Spanish published between January \\ 2007 and May 2018 were selected. \\ Results: Of the 140 articles found, 12 met the inclusion criteria, and were organized into the \\ following three categories: "nurses' competencies in antimicrobials stewardship programs" \\ (five articles), "lack of knowledge and need for training" (six articles), and "systemic action" \\ (one article). \\ Conclusion: Nurses may contribute significantly to antimicrobial stewardship programs; \\ however, educational actions, studies, and public policies are necessary for their effective \\ participation.
}

DESCRIPTORS: Multiple Bacterial Drug Resistance; Nurses; Antimicrobial Stewardship; Professional Competence; Systematic Review.

\section{EL ENFERMERO EN LOS PROGRAMAS DE OPTIMIZACIÓN DEL USO DE ANTIMICROBIANOS: REVISIÓN INTEGRATIVA}

\author{
RESUMEN \\ Objetivo: Investigar publicaciones sobre la participación del enfermero en programas de \\ optimización del uso de antimicrobianos. \\ Método: Revisión integrativa con búsqueda en bases Medical Literature Analysis and \\ Retrieval System on Line, Literatura Latinoamericana en Ciencias de la Salud y Cumulative \\ Index to Nursing and Allied Health Literature. Para inclusión, fueron seleccionados artículos \\ disponibles integralmente, publicados entre enero de 2007 y mayo de 2018, publicados en \\ portugués, inglés o español. \\ Resultados: Se encontraron 140 artículos, 12 atendían los criterios de inclusión. Fueron \\ organizados en tres categorías: "competencias del enfermero en los programas de optimización \\ del uso de antimicrobianos" (cinco artículos), "déficit de conocimiento y necesidad de \\ capacitación" (seis artículos), y "acción sistémica" (un artículo). \\ Conclusión: Los enfermeros pueden contribuir sustancialmente con los Programas de \\ Optimización del Uso de Antimicrobianos, resultando necesarias acciones educativas, \\ investigaciones y políticas públicas para que su participación resulte efectiva.
}

DESCRIPTORES: Farmacorresistencia Bacteriana Múltiple; Enfermeros; Programas de Optimización del Uso de los Antimicrobianos; Competencia Profesional; Revisión Sistemática. 
A resistência microbiana é um problema crescente que limita e dificulta o tratamento das infecções relacionadas à assistência à saúde (IRAS), especialmente em pacientes hospitalizados ${ }^{(1)}$.

Estudos apontam que o uso inadequado de antimicrobianos em hospitais varia entre $25 \%$ e $68 \%$ e resultam no surgimento de microrganismos multirresistentes, IRAS, aumento dos custos associados ao tratamento do paciente, aumento do tempo de internação e aumento da taxas de mortalidade ${ }^{(1)}$. Além disso, estudos apontam que a maior incidência de erros de medicação ocorre durante a administração de antibióticos ${ }^{(2-3)}$.

Otimizar a prescrição de antimicrobianos, monitorar o seu uso e combinar essas ações com estratégias de prevenção e controle de infecção, mostraram-se eficazes em reduzir as IRAS(4). Adicionalmente, a seleção criteriosa de antimicrobianos e a duração adequada da terapia retardam ou evitam o surgimento de microrganismos resistentes ${ }^{(1,4)}$.

O Antimicrobial Stewardiship Program, também conhecido como Programa de Gerenciamento do uso de Antimicrobianos (PGA), surgiu na década de 90, porém o primeiro guia de orientação de como implantá-lo nos serviços de saúde foi publicado pela Infectious Disease Society of America e The Society for Healthcare Epidemiology of America somente em $2007^{(4)}$.

O PGA trata de abordagem multidisciplinar para minimizar o desenvolvimento de microrganismos multirresistentes por meio de uma estratégia multimodal, que contempla a seleção de antimicrobianos apropriados, a otimização da dose e duração, minimizando a toxicidade e os efeitos colaterais ${ }^{(1)}$.

Os PGAs foram implementados em vários hospitais no Brasil e no mundo e, apesar de descritos como multidisciplinares, atualmente há falta de reconhecimento de como os enfermeiros podem participar nos PGAs ${ }^{(1)}$.

Estudo publicado em 2016 descreve a existência de 900 artigos sobre PGAs publicados em revistas médicas, farmacêuticas ou de microbiologia, sendo que apenas 11 publicações foram encontradas em revistas de enfermagem, revelando a falta de literatura sobre o papel dos enfermeiros nos PGAs ${ }^{(5)}$.

Em outro estudo publicado em 2005, os autores citam que a colaboração dos enfermeiros controladores de infecção nesses programas restringe-se ao compartilhamento de dados multidisciplinares, demonstrando que a sua contribuição potencial para o gerenciamento do uso de antimicrobianos permanece sub-explorada ${ }^{(1,6)}$.

No Brasil, a Resolução da Diretoria Colegiada - RDC no 7 de $2010^{(7)}$ e o Programa Nacional de Prevenção e Controle de IRAS (PNCIRAS) - quadriênio 2016-2020(8) recomendam que as instituições de saúde desenvolvam ações para reduzir e controlar a incidêndia de microrganismos multirresistentes, enquanto a Diretriz Nacional para Elaboração de Programa de Gerenciamento do Uso de Antimicrobianos em Serviços de Saúde, publicada pela Agência Nacional de Vigilância Sanitária (ANVISA), descreve as responsabilidades dos membros executores dos PGAs, mas não define claramente o papel do enfermeiro(4).

Neste sentido, esta revisão integrativa buscou trazer contribuições importantes para a enfermagem, para contribuir na construção do conhecimento sobre práticas interdisciplinares que otimizam o uso de antimicrobianos e melhoram a segurança do paciente. Sendo assim, esse estudo tem por objetivo investigar, na literatura, a participação do Enfermeiro nos programas de gerenciamento do uso de antimicrobianos. 
Trata-se de uma Revisão Integrativa de Literatura (RIL), a qual permite a síntese e a análise do conhecimento científico já produzido sobre o tema investigado, propiciando sinalizar lacunas do conhecimento que precisam ser preenchidas com o desenvolvimento de novos estudos ${ }^{(9)}$. A revisão foi conduzida por duas pesquisadoras independentes no período de julho de 2017 a maio de 2018.

As etapas percorridas para a elaboração do presente estudo foram: estabelecimento da questão de pesquisa e objetivos da revisão; critérios de inclusão e exclusão dos artigos (seleção da amostra); categorização dos estudos; análise dos resultados; discussão e apresentação dos resultados e síntese do conhecimento ${ }^{(9)}$.

Para guiar esta revisão, formulou-se a seguinte questão de pesquisa: "Como se dá a participação do enfermeiro nos programas de gerenciamento do uso de antimicrobianos?"

Para responder à questão de pesquisa, foram consultadas três bases de dados da área da saúde: Medical Literature Analysis and Retrieval System on Line (Medline/ Pubmed), Literatura Latino-Americana de Ciências da Saúde (LILACS) e Cumulative Index to Nursing and Allied Health Literature (CINAHL). Os descritores foram definidos de acordo com cada base de dados consultada, sendo o Medical Subject Headings (MeSH) na Medline/Pubmed, os Descritores em Ciências da Saúde (DeCS) na LILACS e List of Headings da CINAHL. Para a busca das publicações em cada uma das bases de dados, os descritores foram combinados pelos operadores boleanos AND/OR. O Quadro 1 descreve a estratégia de busca em cada uma das bases consultadas.

Quadro 1 - Descritores selecionados e estratégia de busca em cada base de dados. São Paulo, SP, Brasil, 2018

\begin{tabular}{|c|c|c|}
\hline \multicolumn{3}{|c|}{ Descritores } \\
\hline Medline/ Pubmed & LILACS & CINAHL \\
\hline ("Anti-infective Agents"[Mesh] & (tw:(Anti-infecciosos)) OR & (MH "Antibiotics+") OR \\
OR anti-infective OR anti- & (tw:(Ação Antimicrobiana)) & "antibiotic" OR (MH "Drug \\
bacterial OR antimicrobial* & OR (tw:(Agentes Anti- & Resistance, Microbial") OR (MH \\
OR antibiotic*) AND & Infecciosos)) OR (tw:(Fármaco & "Antiinfective Agents") OR \\
(stewardship OR "Practice & Anti-Infecciosos)) OR (tw: & "antimicrobial", "stewardship" \\
Patterns, Physicians"[Mesh] & (Microbicidas)) OR (tw: & OR (MH "Prescribing Patterns") \\
OR "prescribing practices" OR & (Agentes Antimicrobianos)) OR & OR "prescribing patterns", \\
"restriction polices") AND nurs* & (tw:(Antimicrobianos)) AND & (MH "Public Policy") OR (MH \\
& "(tw:(Gestão de Antimicrobianos)) & "Hospital Policies") OR (MH \\
& OR (tw:(Gestão de & "Health Policy") OR (MH "Policy \\
& Antibacterianos)) OR(tw:(Gestão & Making") OR (MH "Health Policy \\
& de Antibióticos)) AND (tw:(nurs*)) & Studies") AND "nurse" OR (MH \\
& \multicolumn{2}{|c|}{ "Nurse+") OR "nursing" OR } \\
& \multicolumn{2}{|c|}{ "nurses role". } \\
\hline
\end{tabular}

Os critérios de inclusão para a seleção dos artigos foram: artigos primários, com abordagem qualitativa, quantitativa ou mista, indexados nas bases de dados selecionadas, disponíveis na íntegra; publicados no idioma inglês, português ou espanhol; que contemplavam a participação do enfermeiro no controle da resistência microbiana; publicados no período entre janeiro de 2007 e maio de 2018. A delimitação do período da busca justifica-se pelo fato de que o primeiro guia de orientação para instituir o PGAs foi publicado em $2007^{(4)}$. 
Foram excluídas citações, editoriais, cartas, comentários, resumos de anais, publicações duplicadas, teses, dissertações, trabalho de conclusão de curso, artigos de revisão, livros e capítulos de livros, e artigos que não abordavam a participação do enfermeiro no controle da resistência microbiana.

Na primeira análise, foram lidos os títulos e resumos das publicações, para verificar a adequação aos critérios de inclusão descritos. Nos casos em que o título e o resumo eram insuficientes para definir a temática pesquisada, foi feita a leitura da publicação na íntegra. O fluxo dos artigos selecionados está representado na Figura 1.

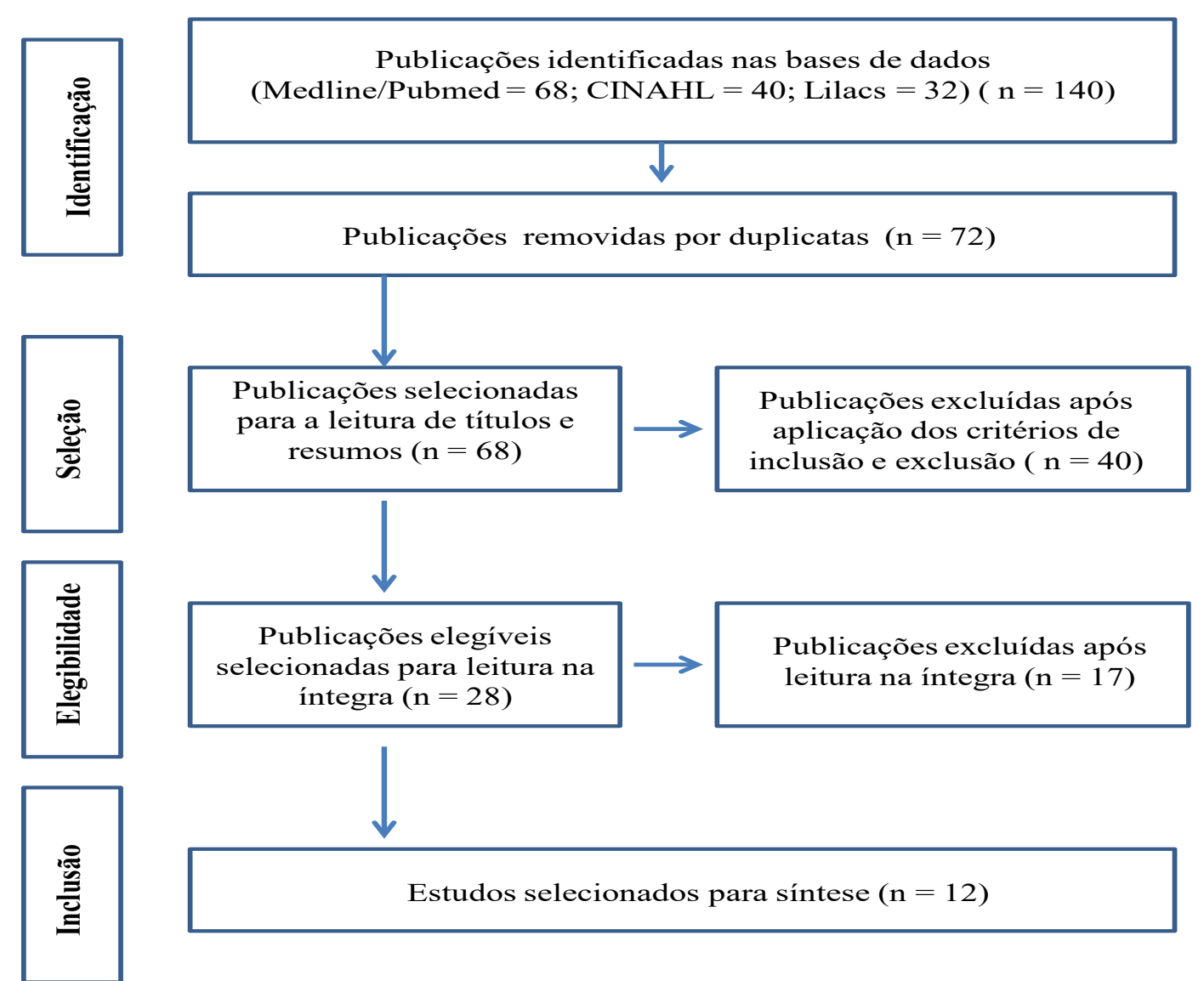

Figura 1 - Diagrama de fluxo dos artigos selecionados, avaliados para elegibilidade, incluídos e excluídos. São Paulo, SP, Brasil, 2018

Os estudos foram classificados segundo o nível de evidência, a saber: Nível 1 evidências de síntese de estudo de coorte ou de estudos de caso controle; Nível 2 evidências de um único estudo de coorte ou estudo de caso controle; Nível 3 - evidências de metassíntese de estudos qualitativos ou de estudos descritivos; Nível 4 - evidências de um único estudo qualitativo ou descritivo; Nível 5 - evidências oriundas de opinião de especialistas ${ }^{(10)}$.

Para a análise e posterior síntese dos artigos que atenderam aos critérios de

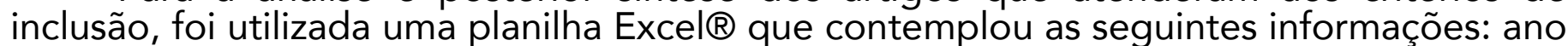
de publicação; autoria; país de origem; delineamento metodológico, principais resultados e nível de evidência. A apresentação dos resultados obtidos foi feita de forma descritiva.

Por se tratar de um estudo que utilizou base de dados de domínio público e por não contemplar a participação de seres humanos como sujeitos de pesquisa, não foi necessária a submissão desse estudo à apreciação de Comitê de Ética em Pesquisa. 
No cruzamento dos descritores foram encontrados 140 artigos que abordaram a participação do enfermeiro nos PGAs. Após leitura dos títulos, resumos e refinamento da busca, foram selecionados 12 artigos, que compuseram a amostra, como apresentado na Tabela 1.

Tabela 1 - Caracterização das publicações sobre a participação do enfermeiro nos Programas de gerenciamento do uso de antimicrobianos. São Paulo, SP, Brasil, 2018

\begin{tabular}{|c|c|c|c|c|}
\hline Ano & Autores & Título do artigo & Periódico & $\begin{array}{l}\text { País de } \\
\text { origem }\end{array}$ \\
\hline 2011 & $\begin{array}{l}\text { Edwards et } \\
\text { al, } 2011^{(1)}\end{array}$ & $\begin{array}{l}\text { Covering more territory to fight resistance: } \\
\text { considering nurses'role in antimicrobial stewardship }\end{array}$ & J Infect Prev & Inglaterra \\
\hline 2012 & $\begin{array}{l}\text { Abbo et al, } \\
2012^{(11)}\end{array}$ & $\begin{array}{l}\text { Nurse practitiones' attitudes, perceptions, and } \\
\text { knowledge about antimicrobial stewardship. }\end{array}$ & $\begin{array}{l}\text { The Journal } \\
\text { of Nurse } \\
\text { Practitioners }\end{array}$ & $\begin{array}{l}\text { Estados } \\
\text { Unidos }\end{array}$ \\
\hline 2013 & $\begin{array}{l}\text { Gillespie et } \\
\mathrm{al}^{(12)}\end{array}$ & Improving antibiotic stewardship by involving nurses. & $\begin{array}{l}\text { American } \\
\text { Journal of } \\
\text { Infection Control }\end{array}$ & Austrália \\
\hline 2014 & $\begin{array}{l}\text { Abera et } \\
\mathrm{al}^{(13)}\end{array}$ & $\begin{array}{l}\text { Knowledge and beliefs on antimicrobial resistance } \\
\text { among physicians and nurses in hospitals in Amhara } \\
\text { Region, Ethiopia. }\end{array}$ & $\begin{array}{l}\text { BMC Pharmacol } \\
\text { Toxicol }\end{array}$ & Etiópia \\
\hline 2015 & $\begin{array}{l}\text { Toska et } \\
\mathrm{al}^{(14)}\end{array}$ & $\begin{array}{l}\text { Antibiotic resistance and irrational prescribing in } \\
\text { paediatric clinics in Greece. }\end{array}$ & Br J Nurs & Grécia \\
\hline 2015 & $\begin{array}{l}\text { Hamilton et } \\
\mathrm{al}^{(15)}\end{array}$ & $\begin{array}{l}\text { Point-of-prescription interventions to improve } \\
\text { antimicrobial stewardship. }\end{array}$ & Clin Infect Dis & $\begin{array}{l}\text { Estados } \\
\text { Unidos }\end{array}$ \\
\hline 2015 & $\begin{array}{l}\text { McGregor } \\
\text { et al }{ }^{(16)}\end{array}$ & Assessing knowledge of antimicrobial stewardship. & Nurs Times & Escócia \\
\hline 2016 & $\begin{array}{l}\text { Manning et } \\
\mathrm{al}^{(17)}\end{array}$ & $\begin{array}{l}\text { Combating antibiotic resistance: the role of nursing in } \\
\text { antibiotic stewardship. }\end{array}$ & $\begin{array}{l}\text { American } \\
\text { Journal of } \\
\text { Infection Control }\end{array}$ & $\begin{array}{l}\text { Estados } \\
\text { Unidos }\end{array}$ \\
\hline 2016 & $\begin{array}{l}\text { Wentzel et } \\
\mathrm{al}^{(18)}\end{array}$ & $\begin{array}{l}\text { Antibiotic information application offers nurses quick } \\
\text { support. }\end{array}$ & $\begin{array}{l}\text { Am J Infect } \\
\text { Control }\end{array}$ & Holanda \\
\hline 2016 & $\begin{array}{l}\text { Oleans et } \\
\mathrm{al}^{(5)}\end{array}$ & $\begin{array}{l}\text { The critical role of the staff nurse in antimicrobial } \\
\text { stewardship - unrecognized, but already there. }\end{array}$ & $\begin{array}{l}\text { Clinical } \\
\text { Infectioous } \\
\text { Diseases }\end{array}$ & $\begin{array}{l}\text { Estados } \\
\text { Unidos }\end{array}$ \\
\hline 2017 & $\begin{array}{l}\text { Wilson et } \\
\text { al }{ }^{(19)}\end{array}$ & $\begin{array}{l}\text { An online course improves nurses' awareness of their } \\
\text { role as antimicrobial stewards in nursing homes. }\end{array}$ & $\begin{array}{l}\text { American } \\
\text { Journal of } \\
\text { Infection Control }\end{array}$ & $\begin{array}{l}\text { Estados } \\
\text { Unidos }\end{array}$ \\
\hline 2018 & $\begin{array}{l}\text { Carter et } \\
\mathrm{al}^{(20)}\end{array}$ & $\begin{array}{l}\text { Exploring the nurse's role in antibiotic stewardship: } \\
\text { a multisite qualitative study of nurses and infection } \\
\text { preventionists }\end{array}$ & $\begin{array}{l}\text { American } \\
\text { Journal of } \\
\text { Infection Control }\end{array}$ & $\begin{array}{l}\text { Estados } \\
\text { Unidos }\end{array}$ \\
\hline
\end{tabular}

Os doze artigos incluídos nesta revisão foram todos publicados no idioma inglês, entre os anos de 2011 a 2018, destacando-se os anos de 2015 e 2016 com seis publicações. Quanto à origem, seis estudos (50\%) foram publicados pelos Estados Unidos, e com relação à formação do autor principal, nota-se que oito estudos $(66,6 \%)$ foram publicados por profissionais de área diferente da enfermagem. 
Com relação à classificação do nível de evidência, oito das publicações $(66,6 \%)$ foram classificadas como nível 4 - evidências de um único estudo descritivo ou qualitativo. Para análise dos dados, os estudos incluídos nesta revisão foram classificados em três categorias: "competências do enfermeiro nos PGAs" (cinco), "déficit de conhecimento e necessidade de capacitação" (seis), "ação sistêmica" (um). O delineamento metodológico, os principais resultados, a categorização dos artigos e o nível de evidência dos estudos são apresentados na Tabela 2.

Tabela 2 - Caracterização das publicações e níveis de evidência dos artigos incluídos na revisão. São Paulo, SP, Brasil, 2018

\begin{tabular}{lll} 
Referência & $\begin{array}{l}\text { Delineamento } \\
\text { metodológico }\end{array}$ & Principais resultados \\
\hline 1 & Descritivo & Os enfermeiros podem participar das \\
& & seguintes etapas do PGAs: especificidade \\
& de tratamento; duração do tratamento; \\
& via de administração do antimicrobiano; \\
& profilaxia cirúrgica; horário da administração; \\
& monitorização terapêutica; terapia \\
& antimicrobiana ambulatorial \\
\hline
\end{tabular}

\section{Categorização Nível de}

Competências do 4

enfermeiro

\begin{tabular}{lll}
\hline 11 & Descritivo & $\begin{array}{l}\text { Os enfermeiros apresentam conhecimento } \\
\text { insuficiente sobre antimicrobianos, o que } \\
\text { reforça a necessidade de uma abordagem } \\
\text { sobre o tema. }\end{array}$ \\
\hline $12 \quad$ Coorte & $\begin{array}{l}\text { Sessões educacionais melhoraram o } \\
\text { conhecimento dos enfermeiros e reduziram o } \\
\text { uso de antimicrobianos administrados por via } \\
\text { endovenosa. }\end{array}$ \\
\hline
\end{tabular}

$13 \quad$ Coorte Os enfermeiros apresentam conhecimento
insuficiente sobre antimicrobianos
Déficit de

conhecimento e

necessidade de

capacitação

Déficit de

conhecimento e

necessidade de

capacitação

Déficit de

conhecimento e

necessidade de

capacitação

Competências do

2

enfermeiro

4

(1)
2

2

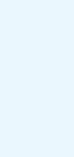

\begin{tabular}{|c|c|c|}
\hline 14 & Coorte & $\begin{array}{l}\text { O envolvimento de enfermeiros nos PGAs } \\
\text { pode contribuir para educar os pacientes } \\
\text { e familiares sobre as implicações do uso } \\
\text { desnecessário de antimicrobianos e da } \\
\text { resistência microbiana à saúde pública. }\end{array}$ \\
\hline
\end{tabular}

15 Descritivo Ação educacional a qual contemplou visita multidisciplinar, auditorias e feedback, melhoraram o conhecimento dos enfermeiros

Déficit de 4

conhecimento e

necessidade de

capacitação

\begin{tabular}{ll}
\hline $16 \quad$ Descritivo & $\begin{array}{l}\text { Os enfermeiros apresentam conhecimento } \\
\text { insuficiente sobre antimicrobianos, e relatam } \\
\text { que a elevada carga de trabalho é uma } \\
\text { barreira para aquisição de conhecimento. }\end{array}$ \\
\hline
\end{tabular}

17 Descritivo Recomenda o uso de ações práticas, educacionais, de pesquisa e políticas públicas

Déficit de

conhecimento e

necessidade de capacitação

Ação sistêmica

4 (ex.: apoio, divulgação e participação em programas nacionais) voltadas ao envolvimento dos enfermeiros nos PGAs

O uso de um aplicativo sobre antimicrobianos foi avaliado positivamente pelos enfermeiros, fornecendo apoio à informação sobre antimicrobianos. 
5 Qualitativo A atuação dos enfermeiros nos PGAs está

Competências do

relacionada a: obtenção de amostras clínicas

enfermeiro

para culturas; interpretação dos resultados

microbiológicos; diferenciação entre

colonização e infecção; avaliação do risco de

infecção e desenvolvimento de uma atitude

questionadora.

\begin{tabular}{lllll}
\hline 19 & Descritivo & $\begin{array}{l}\text { As competências dos enfermeiros nos PGAs } \\
\text { são: avaliação do risco infeccioso, coleta } \\
\text { de amostras laboratoriais para análise } \\
\text { microbiológica e interface com demais } \\
\text { membros da equipe de saúde }\end{array}$ & $\begin{array}{l}\text { Competências do } \\
\text { enfermeiro }\end{array}$ & \\
\hline 20 & Descritivo & $\begin{array}{l}\text { Questionamento sobre a necessidade de } \\
\text { coleta de amostra de urina para cultura, } \\
\text { assegurar a coleta adequada do material } \\
\text { para cultura, encorajar a alteração da via de } \\
\text { administração de endovenosa para oral }\end{array}$ & $\begin{array}{l}\text { Competências do } \\
\text { enfermeiro }\end{array}$ & \\
& &
\end{tabular}

\section{DISCUSSÃO}

Nos Estados Unidos, estima-se que cada ano mais de 2 milhões de pessoas desenvolvam infecção grave causada por bactéria resistente a mais de um antibiótico e pelo menos 23.000 pessoas morrem todos os anos como resultado direto dessas infecções ${ }^{(17)}$. Em média $50 \%$ dos pacientes hospitalizados recebem pelo menos um antibiótico, metade dos quais são desnecessários ou inadequados ${ }^{(19)}$.

Em uma análise retrospectiva de 505 hospitais, 78\% apresentaram evidência de uso potencialmente inapropriados de antibióticos, representando mais de 12 milhões de dólares em custos de cuidados de saúde potencialmente evitáveis. Em serviços ambulatoriais, domiciliares e pediátricos, o uso inapropriado de antimicrobianos acontece em 70\%, 58\% e $10 \%$ dos casos respectivamente $(17,19,21)$.

Segundo estudo realizado em 2016, em serviços de urgência pediátrica, os motivos mais comuns para a prescrição inadequada de antibióticos foram espectro de ação amplo $(41 \%)$, dose incorreta (22\%) e indicação imprópria (17\%)(21). Além disso, estudos apontam maior incidência de erros de medicação durante a administração de antibióticos ${ }^{(2-3)}$.

A resistência aos antimicrobianos é uma das principais ameaças globais de doenças infecciosas, potencialmente comprometedoras para a saúde humana, saúde animal, a agricultura e a economia. Essa crescente resistência foi nomeada pelo Centers for Disease Control and Prevention (CDC) como uma das cinco principais ameaças à saúde nos Estados Unidos e levou ao desenvolvimento do Plano de Ação Nacional ao Combater as Bactérias Resistentes aos Antibióticos, o qual exige o estabelecimento de PGAs em todos os hospitais de cuidados intensivos até $2020^{(5,22)}$.

De acordo com a literatura, os PGAs bem sucedidos exigem o envolvimento ativo de todas as especialidades de saúde para implementar estratégias, iniciativas e ações para reduzir a resistência aos antimicrobianos ${ }^{(4,17)}$.

Embora o PGA seja interdisciplinar e deva contemplar a participação de farmacêuticos, médicos, microbiologistas, controladores de infecções e administradores, os enfermeiros ainda não foram totalmente envolvidos, apesar do seu papel central na administração de antibióticos e sucesso demonstrado na implementação de outras práticas de prevenção e controle de infecção ${ }^{(1,17,19,23)}$. Tal evidência cria um ambiente que limita as instituições de saúde de assumirem uma abordagem verdadeiramente interdisciplinar para prevenir a resistência microbiana ${ }^{(1,17,24)}$. 
De acordo com publicações recentes, os enfermeiros são considerados essenciais nos PGAs em virtude de sua posição central de comunicação, coordenação do cuidado, monitorização do estado do paciente $24 \mathrm{~h} /$ dia, segurança e resposta à terapia antimicrobiana ${ }^{(1,5,24)}$.

Como parte do processo de trabalho, os enfermeiros avaliam a fonte de infecção, coletam e registram dados sobre alergia do paciente, obtêm amostras laboratorais para cultura microbiológica, recebem os resultados laboratoriais e de imagem em primeira mão, instituem precauções específicas quando apropriado, iniciam a terapêutica antimicrobiana, acompanham a evolução do paciente, monitoram reações adversas e fazem a interface com outros membros da equipe ${ }^{(19,24)}$.

$\mathrm{Na}$ fase relativa à especificidade do tratamento, os enfermeiros podem avaliar se o tratamento prescrito está alinhado aos resultados microbiológicos, bem como assegurar que a prescrição de antimicrobianos de amplo espectro seja limitada sempre que possível $\left.\right|^{(1,5,17,19,24)}$. No que se refere à duração do tratamento, o enfermeiro, em colaboração com médicos e farmacêuticos, pode contribuir para que os antimicrobianos sejam prescritos por tempo adequado(1,17,24).

Com relação à via de administração, os enfermeiros podem monitorar as prescrições de antimicrobianos endovenosos e participar de discussões sobre a substituição para administração via oral(1,14,19,24). No que se refere à profilaxia cirúrgica, em colaboração com médicos e farmacêuticos, os enfermeiros podem analisar se os antimicrobianos são prescritos por tempo adequado, garantindo que a medicação seja administrada no momento correto como forma de aumentar a sobrevida do paciente e agilizar a alta hospitalar $(1,5,17,19,24)$.

Os enfermeiros também estão em uma posição-chave para facilitar o encaminhamento do paciente para terapia antimicrobiana ambulatorial, nos casos em que a internação hospitalar for destinada para terapia antimicrobiana prolongada. Esta abordagem próativa do atendimento ao paciente reduz o tempo de permanência no hospital, diminuindo assim o risco de adquirir IRAS e custos associados, permitindo que o paciente continue com o tratamento em domicílio ${ }^{(1,24)}$.

Diante do exposto, nota-se que envolver o enfermeiro nos PGAs pode proporcionar momentos para discutir o tratamento, a indicação e a duração dos antimicrobianos, aumentando ainda mais o gerenciamento multidisciplinar e interdisciplinar do PGAs. Porém, ressalta-se o fato de que para exercer adequadamente a função, os enfermeiros precisam ser capacitados ${ }^{(11-13,15-16,18,24)}$.

De acordo com o documento técnico publicado pela Americam Nurses Association (ANA) e CDC em 2017, os enfermeiros sentem-se inseguros com relação aos seus conhecimentos sobre microbiologia e farmacologia. Para isso, esses órgãos sugerem sessões de treinamento sobre os seguintes tópicos: coleta de amostras laboratoriais para cultura e interpretação de resultados; diferença entre colonização e infecção; comunicação assertiva; critérios de troca de via de administração de antimicrobiano - endovenosa versus oral; e histórico de alergia ${ }^{(24)}$.

Segundo publicações, a participação em treinamentos promove a melhora do conhecimento, do raciocínio crítico e do trabalho em equipe ${ }^{(12,15,18-19)}$.

Estudo realizado em 2017 descreve que os enfermeiros que participaram de um curso online sobre antimicrobianos apresentaram melhora do conhecimento (76\% pré-curso para $86 \%$ pós-curso, $p<0,001)$ e autoconfiança para se envolver nas discussões do PGAs ${ }^{(19)}$. Em estudo realizado nos Países Baixos, o uso de um aplicativo sobre antimicrobianos resultou em melhoria da comunicação entre enfermeiro e médico, e agilidade na tomada de decisão(18). Uma outra pesquisa realizada na Austrália demonstrou que, após uma ação educacional, os enfermeiros foram capazes de articular os riscos do tratamento da terapia antimicrobiana endovenosa e se conscientizaram dos benefícios da mudança da terapia antimicrobiana venosa para a terapia antimicrobiana via oral(12). Adicionalmente, devese considerar a contribuição do enfermeiro como educador de pacientes e familiares, 
fornecendo orientações sobre as implicações do uso desnecessário dos antimicrobianos e suas consequências para a saúde pública ${ }^{(14,24)}$.

$\mathrm{Na}$ última década, organizações nacionais e internacionais abordam a resistência aos antibióticos em relatórios, conferências e publicações em periódicos científicos. Porém, mesmo em meio a toda atenção global, a enfermagem como um todo tem sido relativamente silenciosa sobre a questão da resistência antimicrobiana ${ }^{(17)}$.

Neste sentido, estudos propõem ações nacionais e locais para envolver os enfermeiros na temática. No nível nacional, recomenda-se explorar alternativas de reconhecimento profissional, inserir o tema como pauta de reuniões, priorizar publicações sobre o tema em periódicos de enfermagem, e incluir a temática nos currículos de graduação em enfermagem. No nível local, a proposta é promover treinamentos, envolver os enfermeiros nas visitas multidisciplinares, e enfatizar que o uso racional de antimicrobianos é componente da segurança do paciente ${ }^{(17,24)}$.

\section{CONCLUSÃO}

O uso racional de antimicrobianos é componente integral da segurança do paciente, e os enfermeiros têm contribuições significativas para reduzir a resistência aos antibióticos.

Para atuar efetivamente nos PGAs, o enfermeiro deve desenvolver e aprimorar as competências necessárias para atuar de forma interdisciplinar e efetiva; e para isso, a participação em sessões de treinamento é crucial.

A síntese do conhecimento da presente revisão revela a escassez de literatura nacional sobre o tema, o que reflete excelente oportunidade de investigações no campo da enfermagem e de prevenção e controle de IRAS no Brasil.

\section{REFERÊNCIAS}

1. Edwards R, Drumright LN, Kiernan M, Holmes A. Covering more Territory to Fight Resistance: considering nurses' role in antimicrobial stewardship. J Infect Prev. [Internet]. 2011 [acesso em 10 set 2017]; 12(1). Disponível em: https://www.ncbi.nlm.nih.gov/pmc/articles/PMC3083718/.

2. Pereira FGF, Aquino GA de, Melo GAA, Praxedes CO, Caetano JA. Conformidades e não conformidades no preparo e administração de antibacterianos. Cogitare enferm. [Internet]. 2016 [acesso em 15 set 2017]; 21(5). Disponível em: http://dx.doi.org/10.5380/ce.v21i5.45506.

3. Santos RC dos, Pessalacia JDR, Mata LRF da. Fatores de risco na administração de antimicrobianos pela enfermagem: questões bioéticas. Acta Sci. Health Sci. [Internet]. 2016 [acesso em 03 out 2017]; 38(1). Disponível em: http://dx.doi.org/10.4025/actascihealthsci.v38i1.28505.

4. Ministério da Saúde (BR). Agência Nacional de Vigilância Sanitária (ANVISA). Diretriz Nacional para Elaboração de Programa de Gerenciamento do Uso de Antimicrobianos em Serviços de Saúde. [Internet] Brasília: Ministério da Saúde/ANVISA; 2017. [acesso em 10 out 2017]. Disponível em: http://portal.anvisa. gov.br/documents/33852/271855/Diretriz+Nacional+para+Elabora\%C3\%A7\%C3\%A3o+de+Programa+ de+Gerenciamento+do+Uso+de+Antimicrobianos+em+Servi\%C3\%A7os+de+Sa\%C3\%BAde/667979c27edc-411b-a7e0-49a6448880d4.

5. Olans RN, Olans RD, DeMaria AJr. The critical role of the staff nurse in antimicrobial stewardship - unrecognized, but already there. Clin Infect Dis. [Internet]. 2016 [acesso em 20 out 2017]; 62(1).

Disponível em: https://doi.org/10.1093/cid/civ697.

6. MacDougall C, Polk RE. Antimicrobial stewardship programs in health care systems. Clin Microbiol Rev. [Internet]. 2005 [acesso em 30 out 2017]; 18(4). Disponível: https://doi.org/10.1128/CMR.18.4.638- 
7. Ministério da Saúde (BR). Agência Nacional de Vigilância Sanitária. Resolução n. 7, de 24 de fevereiro de 2010. Dispõe sobre os requisitos mínimos para funcionamento de Unidades de Terapia Intensiva e dá outras providências. Diário Oficial da União, [Internet]. 21 ago 2006 [acesso em 06 nov 2017] Disponível em: http://bvsms.saude.gov.br/bvs/saudelegis/anvisa/2010/res0007 2402 2010.html.

8. Ministério da Saúde (BR). Agência Nacional de Vigilância Sanitária. Gerência Geral de Tecnologia em Serviços de Saúde (GGTES). Programa Nacional de Prevenção e Controle de infecções relacionadas à assistência à saúde (2016-2020). [Internet]. Brasília, 04 de nov. 2016 [acesso em 08 nov 2017] Disponível em: https://www20.anvisa.gov.br/segurancadopaciente/index.php/publicacoes/item/pnpciras-2016-2020.

9. Mendes KDS, Silveira RCCP, Galvão CM. Integrative literature review: a research method to incorporate evidence in health care and nursing. Texto contexto-enferm. [Internet]. 2008 [acesso em 12 nov 2017]; 17(4). Disponível em: http://dx.doi.org/10.1590/S0104-07072008000400018.

10. Melnyk BM, Fineout-Overholt E. Evidence based practice in nursing \& healthcare: a guide to best practice. 3. ed. Philadelphia: Wolters Kluwer; 2014.

11. Abbo L, Smith L, Pereyra M, Wyckoff M, Hooton TM. Nurse practitioners' attitudes, perceptions, and knowledge about antimicrobial stewardship. J Nurse Pract. [Internet]. 2012 [acesso em 15 nov 2017]; 8(5). Disponível em: https://doi.org/10.1016/j.nurpra.2012.01.023.

12. Gillespie E, Rodrigues A, Wright L, Williams N, Stuart RL. Improving antibiotic stewardship by involving nurses. Am J Infect Control [Internet]. 2013 [acesso em 20 nov 2017]; 41(4). Disponível em: https://doi.org/10.1016/j.ajic.2012.04.336.

13. Abera B, Kibret M, Mulu W. Knowledge and beliefs on antimicrobial resistance among physicians and nurses in hospitals in Amhara Region, Ethiopia. BMC Pharmacol Toxicol. [Internet]. 2014 [acesso em 27 nov 2017]; 15(26). Disponível em: https://doi.org/10.1186/2050-6511-15-26.

14. Toska A, Geitona M. Antibiotic resistance and irrational prescribing in paediatric clinics in Greece. Br J Nurs. [Internet]. 2015 [acesso em 10 jan 2018]; 24(1). Disponível em: https://doi.org/10.12968/ bjon.2015.24.1.28.

15. Hamilton KW, Gerber JS, Moehring R, Anderson DJ, Calderwood MS, Han JH, et al. Point-ofprescription interventions to improve antimicrobial stewardship. Clin Infect Dis. [Internet]. 2015 [acesso em 15 jan 2018]; 60(8). Disponível em: https://doi.org/10.1093/cid/civ018.

16. McGregor W, Brailey A, Walker G, Bayne G, Sneddon J, McEwen J. Assessing knowledge of antimicrobial stewardship. Nurs Times. [Internet]. 2015 [acesso em 20 jan 2018]; 111(21). Disponível em: https://www.ncbi.nlm.nih.gov/pubmed/26492697.

17. Manning ML, Pfeiffer J, Larson EL. Combating antibiotic resistance: the role of nursing in antibiotic stewardship. Am J Infect Control. [Internet]. 2016 [acesso em 25 jan 2018]; 44(12). Disponível em: https:// doi.org/10.1016/j.ajic.2016.06.023.

18. Wentzel J, van Drie-Pierik R, Nijdam L, Geesing J, Sanderman R, van Gemert-Pijnen JE. Antibiotic information application offers nurses quick support. Am J Infect Control. [Internet]. 2016 [acesso em 30 jan 2018]; 44(6). Disponível em: https://doi.org/10.1016/j.ajic.2015.12.038.

19. Wilson BM, Shick S, Carter RR, Heath B, Higgins PA, Sychla B, et al. An online course improves nurses' awareness of their role as antimicrobial stewards in nursing homes. Am J Infect Control. [Internet]. 2017 [acesso em 03 fev 2018]; 45(5). Disponível em: https://doi.org/10.1016/j.ajic.2017.01.002.

20. Carter EJ, Greendyke WG, Furuya EY, Srinivasan A, Shelley AN, Bothra A, et al. Exploring the nurses' role in antibiotic stewardship: a multisite qualitative study of nurses and infection preventionists. Am J Infect Control. [Internet]. 2018 [acesso em 06 fev 2018]; 46(5). Disponível em: https://doi.org/10.1016/j. ajic.2017.12.016.

21. Weddle G, Goldman J, Myers A, Newland J. Impact of an educational intervention to improve 
antibiotic prescribing for nurse practitioners in a pediatric urgent care center. J Pediatr Health Care. [Internet]. 2017 [acesso em 08 fev 2018]; 31(2). Disponível em: https://doi.org/10.1016/j. pedhc.2016.07.005.

22. The White House. National action plan for combating antibiotic-resistant bacteria. [Internet]. EUA: The White House; 2015 [acesso em 08 fev 2018]. Disponível em: https://www.cdc.gov/drugresistance/pdf/ national_action_plan_for_combating_antibotic-resistant_bacteria.pdf.

23. Kupfer Y, Savel R. Preventing catheter-associated urinary tract infections. N Engl J Med. [Internet]. 2016 [acesso em 09 fev 2018]; 375(13). Disponível em: https://doi.org/10.1056/NEJMc1609988.

24. American Nurses Association. Centers for Disease Control and Prevention workgroup on the role of registered nurses in Hospital Antibiotic Stewardship Practices. [Internet]. 2017 [acesso em 04 mar 2018]. Disponível em: https://www.cdc.gov/antibiotic-use/healthcare/pdfs/ANA-CDC-whitepaper.pdf.

Recebido: 07/05/2018

Finalizado: 15/02/2019

Autor Correspondente:

Adriana Maria da Silva Felix

Faculdade de Ciências Médicas da Santa Casa de São Paulo

R. Dr. Cesário Mota Júnior, 61 - 01221-020 - São Paulo, SP, Brasil

E-mail: adrianamsfelix1@gmail.com

Contribuição dos autores:

Contribuições substanciais para a concepção ou desenho do estudo; ou a aquisição, análise ou interpretação de dados do estudo - AMSF, SRT

Aprovação da versão final do estudo a ser publicado - AMSF, SRT 